\title{
Development of a Wireless Pressure Transmitter with Diagnostics
}

\author{
Ekaterina Tugova \\ School of Electronic Engineering \\ and Computer Science \\ South Ural State University \\ Chelyabinsk, Russia \\ tugovaes@susu.ru
}

\author{
Manus Patrick Henry \\ University of Oxford, Oxford, UK \\ Coventry University, Coventry, \\ UK. \\ South Ural State University \\ Chelyabinsk, Russia \\ manus.henry@eng.ox.ac.uk
}

\author{
Oleg Bushuev \\ School of Electronic Engineering \\ and Computer Science \\ South Ural State University \\ Chelyabinsk, Russia \\ bushuevoi@susu.ru
}

\author{
Maria Motorina \\ School of Electronic Engineering \\ and Computer Science \\ South Ural State University \\ Chelyabinsk, Russia \\ motorina_maria97@mail.ru
}

\begin{abstract}
The Industrial Internet of Things (IIoT) and Industry 4.0 require new intelligent sensor designs with enhanced functionality, including local diagnostics. In previous work, we have experimentally investigated an important fault mode of a commercial pressure sensor, working in partnership with the sensor manufacturer who has provided modified sensors with calibrated levels of the fault condition. We have further developed simple signal processing techniques to detect the fault condition, based on a low cost noise analysis. In the current paper, we describe the development of a prototype wireless pressure transmitter. This transmitter monitors the analogue output of the pressure sensor, and applies the diagnostic procedures in real time. The resulting pressure measurement in engineering units, together with diagnostic information, are both communicated wirelessly to a receiving system.
\end{abstract}

Keywords - Industrial Internet of Things, fault detection and diagnosis, pressure transducer, self-validation, signal processing, wireless sensors

\section{INTRODUCTION}

The development of new digital technologies applied to industry, including the Industrial Internet of Things (IIoT) [1] and Industrie 4.0 [2], will increase demand for 'intelligent' sensors, with increasingly sophisticated capabilities, to operate in diverse working environments from the Smart City [3] to manufacturing and industrial production. This development provides many technical challenges and opportunities for the measurement and control systems communities.

"As systems become more interconnected and diverse, architects are less able to anticipate and design interactions among components, leaving such issues to be dealt with at runtime. Soon systems will become too massive and complex for even the most skilled system integrators to install, configure, optimize, maintain, and merge [4]."

IBM's concept of Autonomic Computing [4] envisages systems with advanced capabilities, including selfconfiguration, self-optimisation, self-healing and selfprotection. These capabilities require suitable support to be developed across the whole hierarchy, from entire systems down to individual components. Thus:

- Self-optimisation : "[Current] Systems have ... manually set, nonlinear tuning parameters, and their numbers increase with each release [4]", whereas in
Autonomic Computing, "components ... continually seek opportunities to improve their own performance and efficiency [4]"

- Self-healing: "[The Autonomic] System automatically detects, diagnoses and repairs localised software and hardware problems [4]"

- Self-protection: The Autonomic System "uses early warning to anticipate and prevent system-wide failures [4]."

Techniques from Big Data (BD) and Machine Learning (ML) are major enablers, but simply applying such methods "top down" over existing infrastructures will limit potential benefits. In practice, the constraints of communication bandwidth and real-time decision-making may require that "the signal will be processed entirely [at] the Point of Acquisition (PoA) [5]" (i.e. within the sensor), so that a more networked approach is needed. Besides, the availability of technology-specific knowhow (for example, manufacturer's detailed understanding of equipment behaviour, internal diagnostics etc) suggest that BD and ML methods can be applied at every level, and draw on multiple domains of data and expertise. For example, the behaviour of a pressure sensor may be analysed as one component within a particular industrial plant with its unique operating procedure; but the same sensor may also be analysed by its manufacturer as one of many near-identical devices during manufacture, calibration etc. The new techniques must therefore facilitate the transmission of expertise between the domains of the device manufacturer and user.

Applied to sensors, these aspirations align with the concept of the Self-Validating (SEVA) Sensor ([6], a UK National Standard [7,8]), alongside the broadly equivalent notion of "metrological self-check" developed in Russia and elsewhere [9-12]. Here, the sensor (taken to include both the transduction and data processing elements) performs self-diagnostics, but further assesses the quality of its measurement data, applying corrections as required for any detected faults, and provides standardised data quality metrics to assist higher level systems evaluate the usefulness of the measurements for particular tasks. These data quality metrics include an on-line, dynamic assessment of metrological uncertainty. SEVA, originally conceived in the process industry context [6], is applicable to sensor networks generally, for example wireless systems [13]. The NAMUR NE107 standard $[8,14]$, which has been widely 
adopted by the suppliers of process instrumentation, has the less ambitious goal of providing standardised diagnostic messaging for instruments of the same type across multiple vendors.

Pressure sensors, measuring absolute, gauge, or differential pressure, are the most widely used sensing devices deployed in the process industries. Verifying that the measurement data is correct is an essential part of ensuring process safety and efficiency. Techniques for checking the diagnostic state of the sensor are therefore needed.

A key requirement for the development of self-validating instrumentation is the creation of 'realistic' fault modes so that detection strategies based on internal signals can be determined. Where a fault mode leads ultimately to complete loss of measurement function, there is particular interest in capturing the transient behaviour that is indicative of the condition so that maintenance action can be taken ahead of measurement failure.

In previous work, we have investigated, both theoretically and experimentally, an important fault mode of a commercial pressure sensor - leakage of the internal working fluid working in partnership with a sensor manufacturer. The manufacturer was able to provide modified sensors with calibrated levels of fluid loss. We have further developed simple signal processing techniques to detect this fault condition, based on a low cost noise analysis. In the current paper, we describe the development of a prototype wireless pressure transmitter. This transmitter monitors the analogue output of the pressure sensor, and applies the diagnostic procedures in real time. The resulting pressure measurement, in engineering units, and diagnostic information are communicated wirelessly to a receiving system.

The demonstration system is based around an STM32 microcontroller, which performs the local measurement and diagnostic calculations, and acts as the wireless transmitter. A host computer receives the data and provides a graphical user interface via an app - a similar app could be used by a process operator using a mobile phone. Experimental work using two different test procedures, with different levels of background noise, demonstrate the utility of the system, while illustrating the need for further work on signal discrimination.

\section{PRESSURE SENSOR DIAGNOSTICS}

Prior research into industrial pressure sensor diagnostics (e.g. [15-22]) supports the assumption that internal signal analysis can be useful in providing timely diagnostics of important fault modes. Each fault detection technique needs to match the signal characteristics of the corresponding fault mode for the particular type of pressure sensing technology. For example, a survey of pressure sensor faults found in industrial applications ([16]) found that the most common reason for failure is damage to the process diaphragm caused by hydraulic shock or rapid pressure pulsations. These pressure pulses result in micro-fractures which, in a typical industrial environment containing corrosive materials, can cause pitting of the protective membrane. This in turn may result in loss of the silicone oil used to transmit the external pressure to the internal transducers, causing measurement error. Earlier research by the current authors, including a companion paper at this conference [23-27]), has examined aspects of this fault mode, including the development of mathematical models of its physical behaviour, predicting the signal characteristics of the fault, developing proposed diagnostic techniques, and carrying out experimental work on sensors with calibrated levels of oil loss, in order to provide verification of the proposed diagnostic techniques.

Ideally, a fault detection technique should be entirely independent of the characteristics of the measurement signal itself. This reduces the risk that any change in the actual process, for example in the measurement noise characteristics, is misinterpreted as a fault symptom. Typically [6], such fault detection entails the identification of additional signals within the transducer, or specially developed device-specific tests, which provide measurement-independent diagnostic information. For example, the current authors have developed a diagnostic technique for pressure sensors, whereby the mechanical integrity of the sensor housing can be checked by use of an internal ultrasonic transmit/receive transducer [23, $26,27]$. Here the frequencies and decay characteristics of the returned signal provide information on the structural integrity of the sensor, irrespective of process behavior, and independently of the pressure measurement signal path.

In practice, the inclusion of such additional transducers, with associated test procedures and computational requirements, may not be economically viable for commercial devices, and the majority of pressure sensor diagnostic procedures reported in the literature are based upon observed characteristics within the measurement signal itself, despite entailing a degree of dependency on process behaviour.

A particular difficulty arises when the fault mechanism is not strictly internal to the sensor, but occurs at the interface between the sensor and the process [6]. Interfacial errors are in general more difficult to diagnose, because the sensor manufacturer has less control or knowledge of the condition of the process interface. A well-known example of an interface fault for pressure sensors is impulse line clogging, where material builds up in the lines connecting the process to the pressure sensor. Hashemian $[16,17]$ investigated how the response time of the pressure measurement depends on the condition of the impulse line. The technique exploited pressure fluctuations inherent in the external industrial process to perform noise analysis, and entailed learning the noise characteristics of the process in order to detect aberration that might be indicative of the fault.

Our companion paper at this conference [25], provides a review of the various types of pressure sensors conventionally used in industrial practice, and considers the main fault modes of the particular technology investigated here, the tensoresistive pressure sensor. Of these various faults, the loss of fill liquid is described in detail, and a mathematical model is developed to describe the expected measurement behavior of the sensor, with simulation results illustrating the results. Figure (1), taken from [25], shows a simplified depiction of the pressure transducer structure, and illustrates the liquid loss fault. Essentially, the pressure sensor consists of an external, relatively soft plate, called the membrane, which is exposed to 


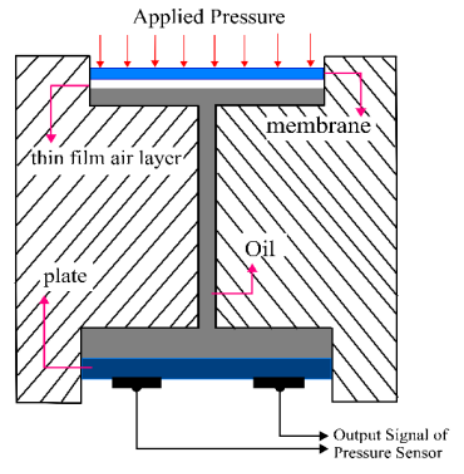

Fig. 1. A schematic view of the cross-section of the tenso-resistive pressure transducer (from [25]).

the pressure source from the industrial process. A second, stiffer plate, here simply labeled 'plate', supports the strain gauge transducers, which are protected inside the pressure sensor housing. The transducer resistance varies with the strain applied to the plate, enabling the external pressure to be determined. The transmission of the external pressure at the membrane to the plate is achieved via an incompressible liquid, typically silicone oil, which fills the channel between the two plates. Where the oil-loss fault occurs, for example as a result of damage to the external membrane, this results in air or other gas forming in the liquid channel; in [25], for simplicity, this is modelled as a thin film of air. The presence of this air interferes with the pressure transmission between the two plates. One, perhaps unexpected, consequence of this fault, but predicted by the mathematical model and confirmed by our experimental work with real devices [24], is that it causes a reduction in the upper limit on the reported pressure output. In other words, the fault lowers the pressure at which the sensor output saturates. This is potentially very serious for any industrial application where the pressure sensor is being used to detect high pressure in the process: once the saturation point is reached, the measurement output will not increase, no matter how high the true process pressure rises.

As reported in [24], experiments were carried out on a set of commercial transducers where the manufacturer adapted the conventional manufacturing procedure to introduce a calibrated volume of air into each transducer. Based on the signal characteristics observed, including the standard deviation, skewness, and power spectral density of the raw transducer data, a simple set of diagnostic rules were developed to determine the sensor's state, as illustrated in Figure 2. Here we report on a prototype wireless-based system which implements this algorithm on-line.

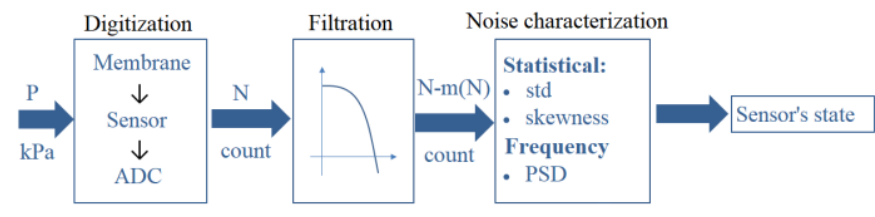

Fig. 2. Pressure transducer signal processing to determine diagnostic state (from [24]).

\section{WIRELESS PROTOTYPE PRESSURE TRANSMITTER}

Figure 3 shows the hardware used in the prototype pressure transmitter. The strain gauge signal from the commercial pressure transducer is amplified by suitable analog gain circuitry and passed to the stm32 Discovery F3 microcontroller, which samples the data using a 12-bit analogto-digital converter (ADC), sampling at $1 \mathrm{kHz}$. The microcontroller then performs local data processing, implementing conversion into to engineering units, data analysis, and diagnostics. The results are transmitted wirelessly to a mobile-phone based application for display. In addition, high frequency raw ADC data can be sent via the app to facilitate off-line analysis, as reported here. Figure 4 shows typical screen displays from the mobile phone-based app.

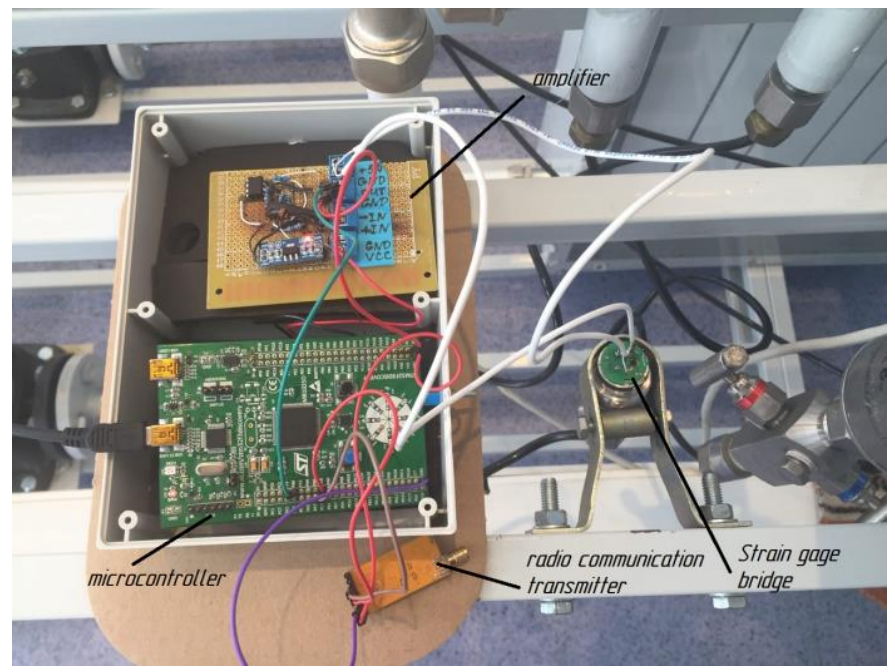

Fig. 3. Prototype transmitter hardware
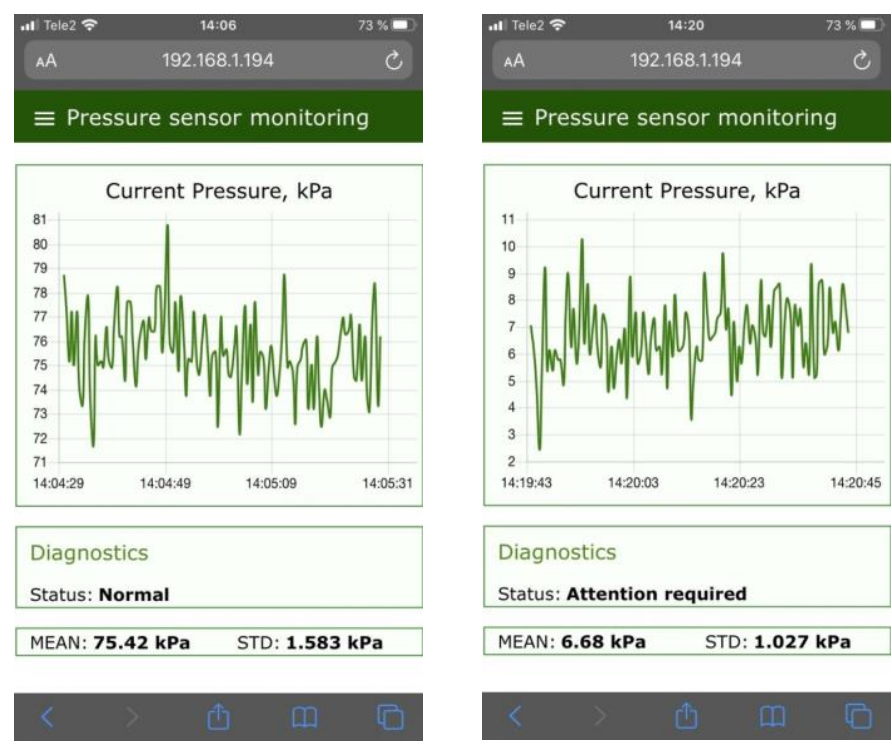

Diagnostics

Status: Attention required

MEAN: $6.68 \mathrm{kPa} \quad$ STD: $1.027 \mathbf{~ k P a}$

Fig. 4. Mobile phone-based app showing pressure sensor measurement and diagnostic output: (left) fault free; (right) fault detected. 
For example, Figure 5 shows typical raw 12-bit ADC output from a fault free pressure transducer over a 60 second period where the input (gauge) pressure is $75 \mathrm{kPa}$. From this raw data, a number of statistics are calculated, including the running mean and standard deviation, from which the measurement and diagnostic state are derived.

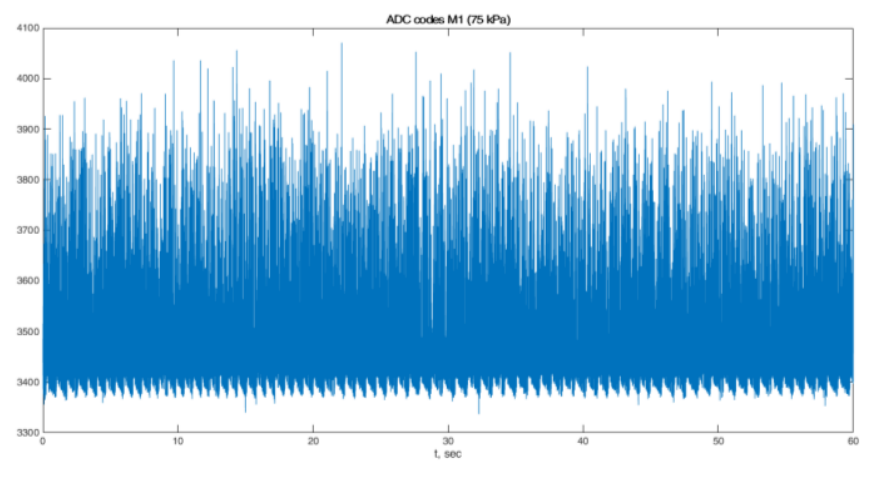

Fig. 5. Typical raw ADC data

\section{EXPERIMENTAL RESULTS}

A simple experimental programme has been carried out to verify the proper functioning of the wireless prototype system, and to explore the impact of using the diagnostic algorithms with low and moderate levels of background noise.

Table I gives the characteristics of the three pressure transducers which were coupled to the prototype transmitter during the experimental work. All were of the same commercial model type: Metran 150 TG2. Unit M1 was a conventional fault-free device, while units M9 and M10 had been modified by the manufacturer so that $46 \%$ of the silicone oil had been removed from the device. This adjustment was applied during manufacturer, so the sensors were otherwise undamaged.

Each transducer was tested over a range of input pressures $(0$, 25 and $75 \mathrm{kPa}$ ) and in two different pressure testing systems. The low noise test system consisted of a portable Metran 501PKD-R pressure calibrator with an h-2.5 pneumatic hand pump (Figure 6). Here the hand pump is used to generate the desired pressure, while the calibrator provides an independent and calibrated reference pressure measurement. In the absence of any active mechanical components, the process noise in this system is generally low, and the pressure is effectively static. The second test system used was the Emerson laboratory facility at South Ural State University (Figure 7). This consists of a water flow loop, with a range of commercial sensors and

TABLE I. PRESSURE TRANSDUCERS UNDER TEST

\begin{tabular}{l|c}
\hline \hline \multicolumn{1}{c|}{ Identifier } & Fluid Loss Condition \\
\hline M1 & $0 \%$ fluid loss \\
\hline M9 & $46 \%$ fluid loss \\
\hline M10 & $46 \%$ fluid loss \\
\hline
\end{tabular}

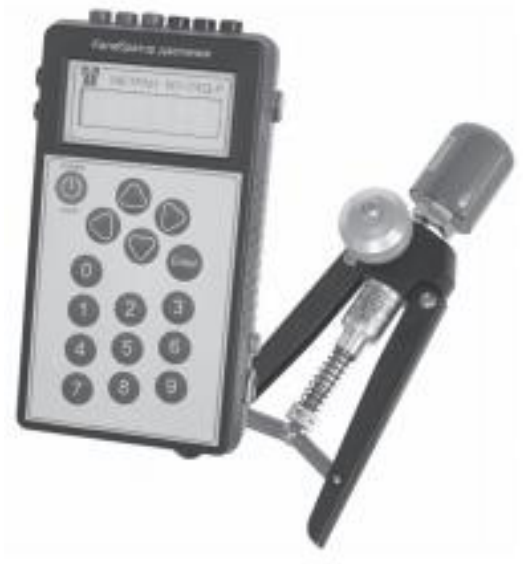

Fig. 6. Portable Metran 501-PCD-R pressure Calibrator

actuators. In this experiment, the actuators used were a pump and a control valve. The reference measurement was provided by a calibrated Rosemount $3051 \mathrm{~S}$ TG pressure sensor. The selected pressure set point was maintained using a PID control algorithm. This system provides moderate levels of measurement noise, arising from the activity of (at least) the pump and valve.

Figures $8-15$ show results obtained from the experimental work. The first four figures are obtained with the pressure calibrator, while the second set are the equivalent results obtained in the Emerson Lab. Results are plotted for each transducer (M1, M9 or M10), for each of three pressure points $(0,25,75 \mathrm{kPa})$. For each lab, the following results are plotted:

- Mean ADC values with offset removed. The Wheatstone bridge voltage from the transducer has a zero offset, which is transducer-specific. The corresponding ADC values have a similar offset at zero input pressure. This is recorded as a device-specific calibration constant, and subtracted before further calculations proceed.

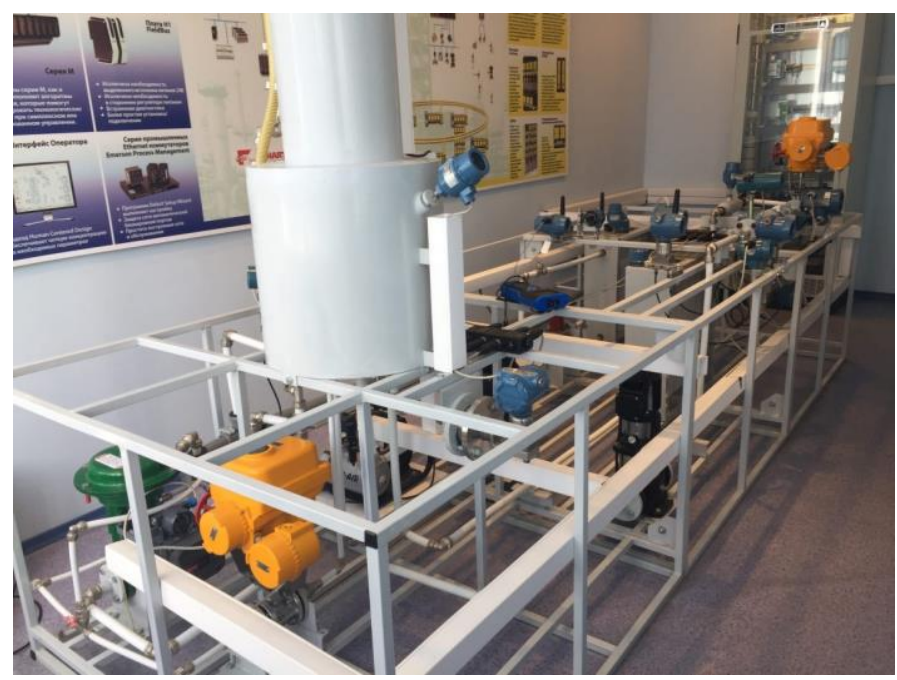

Fig. 7. Emerson flow test facility 


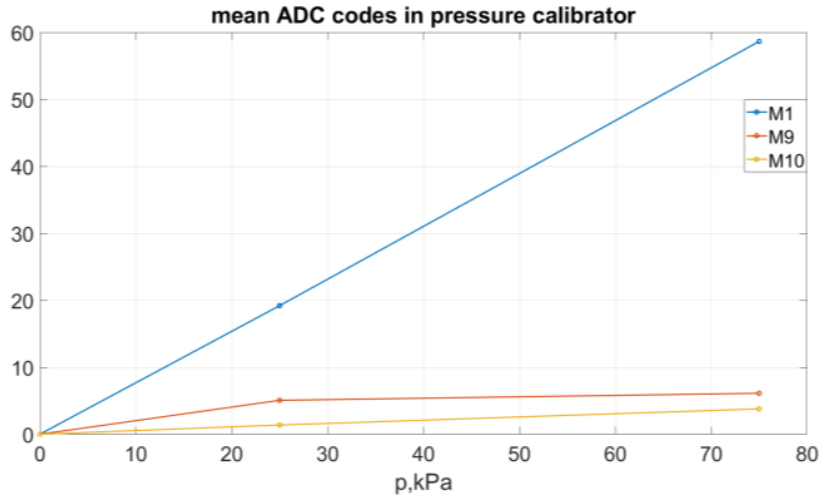

Fig. 8. Mean ADC values, offset removed - pressure calibrator

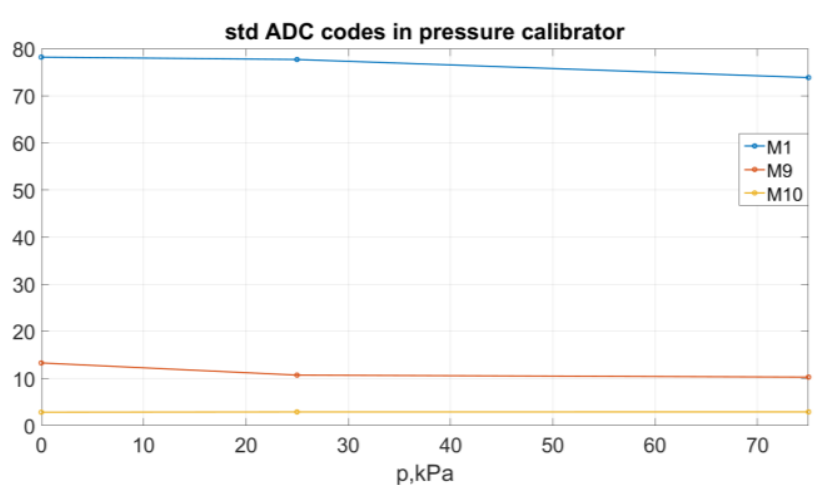

Fig. 9. Standard deviation of ADC values- pressure calibrator

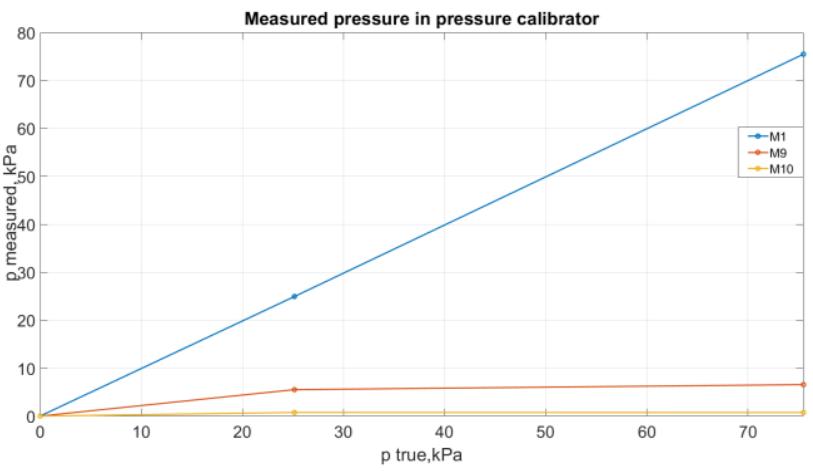

Fig. 10. Measured pressure - pressure calibrator

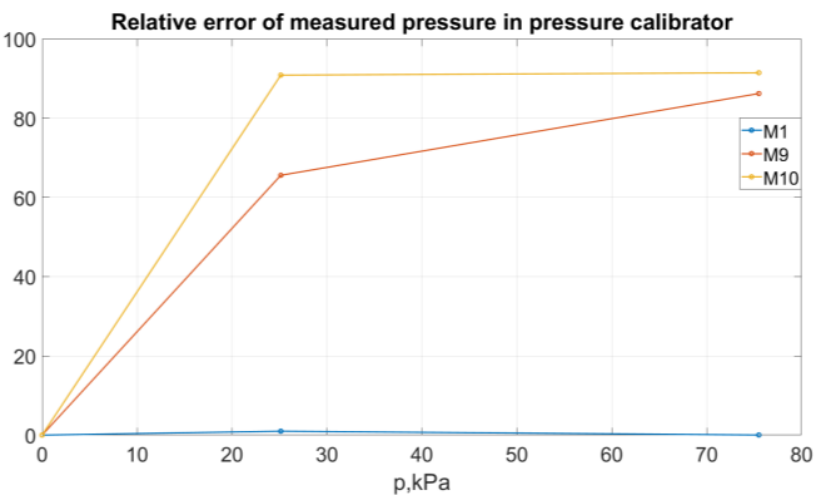

Fig. 11. Relative error in measured pressure - pressure calibrator

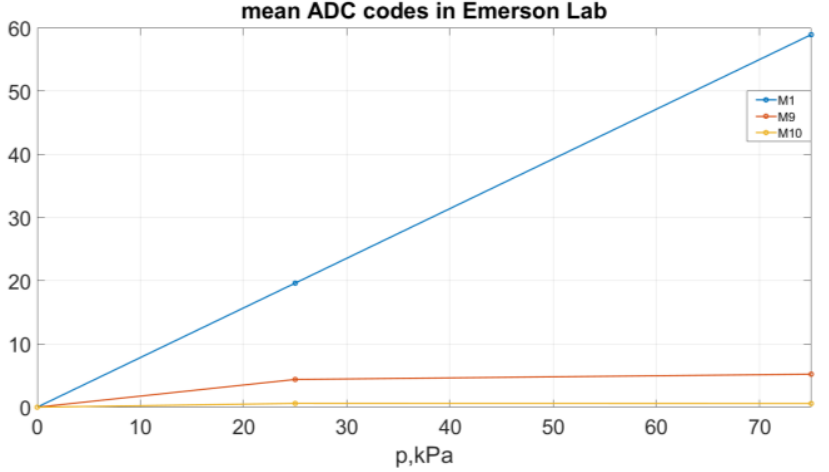

Fig. 12. Mean ADC values, offset removed - Emerson Lab

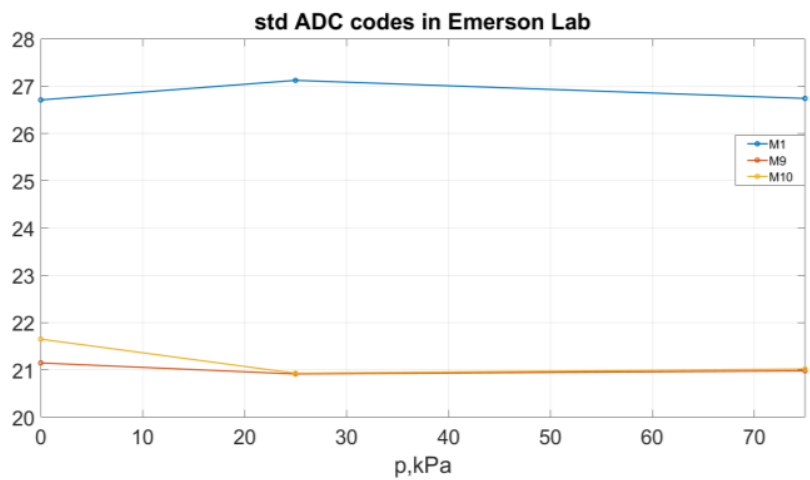

Fig. 13. Standard deviation of ADC values- Emerson Lab

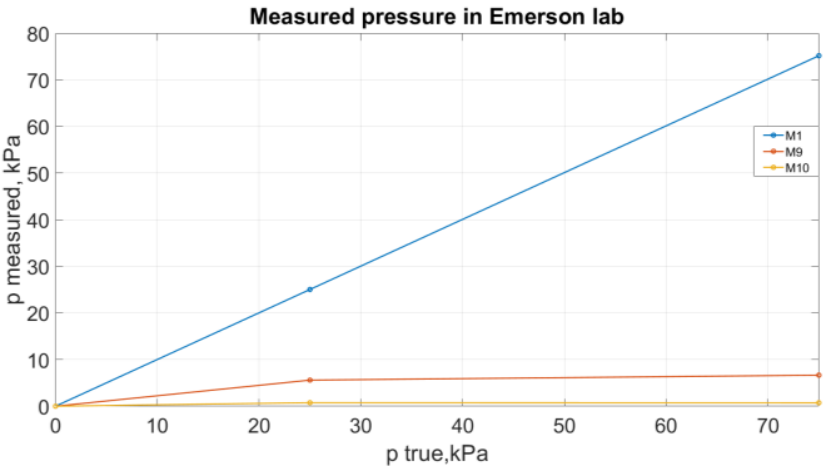

Fig. 14. Measured pressure - Emerson Lab

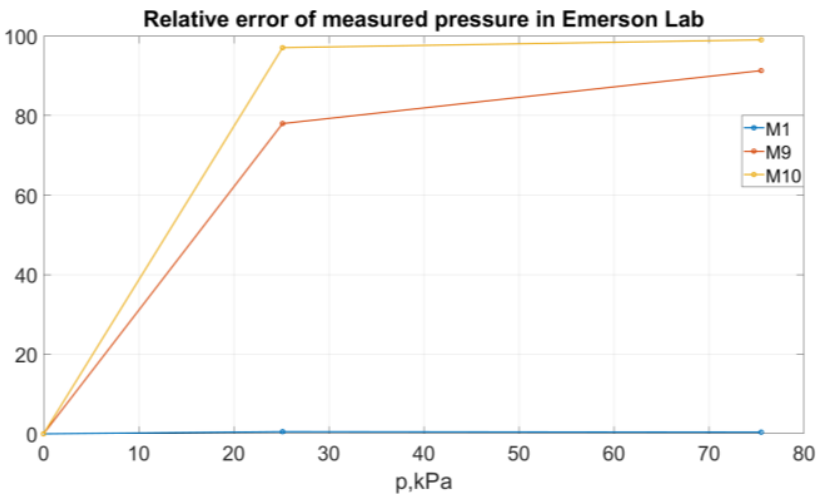

Fig. 15. Relative error in measured pressure - Emerson Lab 
- Standard deviation of the ADC values. For each experimental point, the standard deviation is calculated over the $60 \mathrm{~s}$ of experimental data, sampled at $1 \mathrm{kHz}$. Note that while the standard deviation for individual samples is high (compared to the mean values), averaging over a typical measurement reporting period (e.g. 0.1 1s) will significantly reduce the level of noise.

- Output pressure measurement, in $\mathrm{kPa}$. This is essentially a linear function of the offsetcorrected ADC mean value, where the slope is a second calibration coefficient unique to each transducer. The current system does not take into account temperature effects in its pressure calculation.

- Relative pressure error, expressed as a percentage.

The results obtained from the wireless prototype system match the earlier findings, namely that the fault free sensor (M1) shows good linearity (Figures $10 \& 14$ ), with low errors (Figures $11 \& 15$ ) operating in both the pressure calibrator and the Emerson Lab. By contrast, the faulty transducers M9 and M10 exhibit output saturation for both test stands (Figures 8, 12 ), leading to large errors (Figures $11 \& 15$ ).

The standard deviation results (Figures $9 \& 13$ ) are more complex, and demonstrate the need for further analysis. As expected, the observed standard deviations are significantly lower for the faulty transducers than for the fault free sensor, supporting the use of standard deviation as a diagnostic. Also as expected, the noise level in the Emerson Lab is generally higher than for the pressure calibrator. In the case of the Emerson Lab (Figure 13), the reduction in noise from the fault free to the faulty case ( $\sim 27$ to around $\sim 21)$ is relatively small, so that detecting liquid loss based solely on a learned threshold value for the std might be subject to false alarms should the process noise characteristic itself change over time.

[*** Note to reviewers: the std results for the pressure calibrator (Figure 9) include unexpectedly high values for the fault free case. We suspect electronic noise is responsible our ability to perform further experimental work has been limited in recent months. All these experimental results will be repeated (with additional intermediary pressure values) in the final version of the paper, with the analysis adjusted accordingly.]

\section{DISCUSSION AND CONCLUSIONS}

This paper has summarized prior work in the development of validation for pressure sensors, in particular for the detection of silicone oil leakage. A new prototype transmitter has been developed which operates in conjunction with a commercial pressure transducer, which is able to perform basic measurement functions, but also implements a simple diagnostic test to detect oil leakage, whereby both the measurement and diagnostic data is reported via mobile phone based app. The prototype system has been tested in two different facilities, one with low process noise, and another with moderate process noise. The variation in noise properties, while not preventing accurate diagnosis in this example, demonstrates the importance of reducing the dependence of diagnostic techniques upon learned process noise characteristics.

In future work, we will:

- develop a new test stand allowing the introduction of measurement noise with desired spectral properties, to develop more robust modelling, analysis and detection of the signal characteristics of the liquid loss condition;

- develop new experimental procedures to provide controlled degradation of the sensor structure (e.g. via fatigue); this will complement the analysis of controlled liquid leakage.

\section{ACKNOWLEDGMENT}

The team thanks "METRAN IG", as well as the Aerospace Department of SUSU for support and the provision of equipment for conducting experiments.

\section{REFERENCES}

[1] L.D. Xu, W. He, and S.L. Wang, "Internet of Things in Industries: A Survey", IEEE Transactions on Industrial Informatics, vol. 10, No. 4, pp. 2233-2243, Nov. 2014. DOI :10.1109/TII.2014.2300753.

[2] B. Vogel-Heuser, D. Hess, "Guest Editorial - Industry 4.0-Prerequisites and Visions", IEEE Transactions on Automation Science and Engineering, $\quad \mathrm{Vol}$ 14, No. 2, April 2016. DOI: 10.1109/TASE.2016.2523639.

[3] Zanella et al, "Internet of Things for Smart Cities", IEEE Internet of Things Journal, Feb 2014.

[4] J. O. Kephart and D. M. Chess, "The vision of autonomic computing", IEEE Computer, vol. 36, no. 1, pp. 41-50, Jan. 2003.

[5] G. Monte, V. Huang, P. Liscovsky, D. Marasco, and A. Agnello,"Standard of things, first step: Understanding and normalizing sensor signals," Industrial Electronics Society, IECON 2013 - 39th Annual Conference of the IEEE, Vienna, 2013, pp. 118-123. DOI: 10.1109/IECON.2013.6699121

[6] M.P. Henry and D.W. Clarke, "The Self-Validating Sensor: Rationale, Definitions and Examples", Control Eng. Pract., vol. 1, pp 585-610, 1993.

[7] BSI, "BS7986:2005, Specification for data quality metrics for industrial measurement and control systems", British Standards Institute, 2005.

[8] M.P. Henry and G.G. Wood, "Sensor Validation: principles and standards", atp International 3, No. 2, pp 39-52, 2005.

[9] Taymanov R.E., Sapozhnikova K.V. "Metrological self-check and evolution of metrology", Measuremen, vol.t 43(7), pp. 869-877.

[10] GOST R 8.615, Amended 2008. "State system for ensuring uniformity of measurements. Measurement of quantity of oil and petroleum gas extracted from subsoil. General metrological and technical requirements". Federal Agency for technical regulation and metrology. 2008. (in Russian).

[11] $R$. Taymanov, K. Sapozhnikova, I. Druzhinin, "Sensor Devices with Metrological Self-Check", Sensors \& Transducers Journal, Vol. 10, pp. 30-45, February 2011.

[12] R. Taymanov, K. Sapozhnikova, I. Danilova, I. Druzhinin, "MultiChannel Intelligent Measuring Systems", XXI IMEKO World Congress, Aug 30 - Sep 4, 2015, Prague, Czech Republic.

[13] M. A. Collett, M. G. Cox, M.D. Duta, T.J. Esward, P.M. Harris and M.P. Henry, "The application of self-validation to wireless sensor 
networks", Meas. Sci. Technol. vol 19, 2008. DOI:10.1088/09570233/19/12/125201

[14] NE107 "Self-Monitoring and Diagnosis of Field Devices", NAMUR, Version: June 2006.

[15] Blazquez, J. and Ballestrin J. (1995) 'Pressure transmitter surveillance: the dominant real pole case', Progress in Nuclear Energy, Vol. 29, No. 3, pp. 290-303.

[16] Hashemian, H.M., Mitchell, D.W., Fain, R.E. and Petersen, K.M. "Long Term Performance and Aging Characteristics of Nuclear Plants Pressure Transmitters", Nuclear Regulatory Commission NUREG/CR5851, 1993, pp.70-79, pp.290-293

[17] Hashemian, H. M. Measurement of Dynamic Temperatures and Pressures in Nuclear Power Plants // Electronic Thesis and Dissertation Repository. Paper 189. 2011.

[18] Wehrs, D. Detection of Plugged Impulse Lines Using Statistical Process Monitoring Technology / D. Wehrs. Emerson Process Management. 2006.

[19] Barbero, J., Blázquez, J. and Vela O.'Bubbles in the sensing line of nuclear power plant pressure transmitters: the shift of spectrum resonances', Nuclear Engineering and Design, 2000, Vol. 199, pp. 327334.

[20] Barbero, J., Blázquez, J. and Vela O. 'Bubbles in the sensing line of nuclear power plant pressure transmitters: the shift of spectrum resonances', Nuclear Engineering and Design, 2000, Vol. 199, pp. 327334.

[21] García-Berrocal, A., Chicharro, J.M., Blázquez, J. and Balbás, M. 'Nonlinear noise analysis from a capacitive pressure transmitter', Mechanical Systems and Signal Processing, 2004, Vol. 18, pp.187-197.
[22] JB Coble, HM Hashemian, RM Meyer, BD Shumaker, P Ramuhalli, DS Cummins, LJ Bond, 'A Review of Sensor Calibration Monitoring for Calibration Interval Extension in Nuclear Power Plants', U.S. Department of Energy Report PNNL-21687, 2012

[23] Henry, M, Bushuev O, Salov D. , 'Sensor Validation Via Ultrasonic Signal Processing Analysis', 2018 Global Smart Industry Conference (GloSIC), Chelyabinsk, Russia.

[24] Tugova, E, Salov D., Henry, M, Bushuev O. Y. 'Diagnostics of a pressure sensor based on output signal noise characteristics' 2020, International Conference on Industrial Engineering, Russia

[25] Soltani, K, Henry, M, Ghanbari, M, Tugova, E, Bushuev, O, Rezazadeh, G. (2020) 'Investigating Fluid Loss Faults in Piezoresistive Pressure Sensors', 2020 Global Smart Industry Conference, Chelyabinsk, Russia.

[26] O.Yu. Bushuev, A.S. Semenov, A.O. Chernavskiy and A.L. Shestakov, "Detecting changes in the condition of a pressure transucer by analysing its output signal", XX IMEKO World Congress, Busan, 2012.

[27] Henry, MP, Bushuev, O, Ibryaeva, O. "Prism Signal Processing for Sensor Condition Monitoring" . IEEE International Conference on Industrial Electronics, Edinburgh, UK, 2017 